\title{
Genome sequences published outside of Standards in Genomic Sciences, January - June 2011
}

\author{
Oranmiyan W. Nelson ${ }^{1}$ and George M. Garrity ${ }^{1}$ \\ 'Editorial Office, Standards in Genomic Sciences and Department of Microbiology, \\ Michigan State University, East Lansing, MI, USA
}

The purpose of this table is to provide the community with a citable record of publications of ongoing genome sequencing projects that have led to a publication in the scientific literature. While our goal is to make the list complete, there is no guarantee that we may have omitted one or more publications appearing in this time frame. Readers and authors who wish to have publications added to this subsequent versions of this list are invited to provide the bibliometric data for such references to the SIGS editorial office.

\section{Phylum Crenarchaeota}

"Metallosphaera cuprina" Ar-4, sequence accession CP002656 [1]

Thermoproteus uzoniensis 768-20, sequence accession CP002590 [2]

"Vulcanisaeta moutnovskia" 768-28, sequence accession CP002529 [3]

\section{Phylum Euryarchaeota}

Methanosaeta concilii, sequence accession CP002565 (chromosome), CP002566

(plasmid) [4]

Pyrococcus sp. NA2, sequence accession CP002670 [5]

Thermococcus barophilus MP, sequence accession CP002372 (chromosome) and

CP002373 plasmid) [6]

\section{Phylum Chloroflexi}

Oscillochloris trichoides DG-6, sequence accession ADVR00000000 [7]

\section{Phylum Proteobacteria}

Achromobacter xylosoxidans A8, sequence accession CP002287 (chromosome),

CP002288 (plasmid pA81), and CP002289 (plasmid pA82) [8]

Acinetobacter baumannii 3990, sequence accession AEOY00000000 [9]

Acinetobacter baumannii ST78, sequence accession AEPA00000000 [9]

Acinetobacter baumannii, sequence accession AEPA00000000 [9]

Acinetobacter baumannii MDR-TJ, sequence accession AEOE00000000 [10]

Acinetobacter baumannii TCDC-AB0715, sequence accession CP002522 (chromosome), CP002523 (p1ABTCDC0715) and CP002524 (p2ABTCDC0715) [11]

Salmonella enterica serovar Typhimurium UK-1 ATCC 68169, sequence accession CP002614 (chromosome), CP002615 (plasmid) [12]

Acinetobacter calcoaceticus PHEA-2, sequence accession CP002177 [13]

Aeromonas caviae Ae398, sequence accession CACP01000001 to CACP01000149 [14] 
Aeromonas veronii B565, sequence accession CP002607 [15]

Bordetella pertussis CS, sequence accession CP002695 [16]

Brucella melitensis M111, sequence accession AFFB00000000 [17]

Brucella melitensis M28-12, sequence accession AFFA00000000 [17]

Brucella melitensis M5, sequence accession AFEZ00000000 [17]

Brucella suis S2, sequence accession AFFC00000000 [17]

Brucella melitensis M5-90, sequence accession CP001851 and CP001852 [18]

Brucella melitensis M28, sequence accession CP002459 and CP002460 [18]

Burkholderia gladioli BSR3, sequence accession CP002599 to CP002604 [19]

Burkholderia phytofirmans, sequence accession CP001052 to CP001054 [20]

Burkholderia rhizoxinica HKI 0454, sequence accession FR687359 (chromosome), FR687360 (pBRH01), FR687361 (pBRH02) [21]

Campylobacter jejuni S3, sequence accession CP001960 (chromosome) CP001961 (plasmid) [22]

Candidatus Liberibacter solanacearum, sequence accession CP002371 [23]

“Citromicrobium sp." JLT1363, sequence accession AEUE01000000 [24]

Cronobacter turicensis LMG 23827, sequence accession NC_013282 to NC_013285 [25]

Desulfovibrio africanus Walvis Bay, sequence accession AFHE00000000 [26]

Desulfovibrio desulfuricans ND132, sequence accession AEUJ00000000 [27]

Dickeya dadantii 3937, sequence accession CP002038 [28]

“Enterobacter mori” LMG 25706, sequence accession AEXB01000000 [29]

Erwinia amylovora, sequence accession [30]

Erwinia sp. Ejp617, sequence accession CP002124 (chromosome), CP002125 (pJE01), CP002126 (pJE02), CP002127 (pJE03), CP002128 (pJE04), and CP002129 (pJE05) [31]

Escherichia coli AA86 KACC15541, sequence accession AFET00000000 [32]

Escherichia coli UM146, sequence accession CP002167 [33]

“Gallibacterium anati” UMN179, sequence accession CP002667, CP002668 [34]

Gluconacetobacter sp. SXCC-1, sequence accession AFCH0000000 [35]

Haemophilus para SH0165 serovar 5, sequence accession CP001321 [36]

Herbaspirillum seropedicae SmR1, sequence accession [37]

Ketogulonicigenium vulgare Y25, sequence accession CP002224 (chromosome), CP002225 (plasmid), and CP002226 (plasmid) [38]

Methylocystis sp. sp. Rockwell, sequence accession AEVM00000000 [39]

Methylophaga thiooxydans DSM010, sequence accession ABXT00000000 [40] 
Methylovorus sp. MP688, sequence accession CP002258 [41]

Neisseria gonorrhoeae TCDC-NG08107, sequence accession CP002440 and CP002441 [42]

Neisseria meningitidis H44/76, sequence accession AEQZ00000000 [43]

Neisseria meningitidis WUE2594, sequence accession FR774048 [44]

Oceanicaulis sp. HTCC2633, sequence accession AAMQ00000000 [45]

Paracoccus sp. sp. TRP, sequence accession AEPN00000000 [46]

Parvularcula bermudensis HTCC2503T , sequence accession CP002156 [47]

"Photobacterium mandapamensis" svers. 1.1, sequence accession BACE01000001 to BACE01000031 [48]

“Polymorphum gilvum" SL003B-26A1T LMG 25793T, sequence accession CP002568, CP002569 [49]

Pseudomonas savastanoi pv. glycinea (Psg) B076, sequence accession AEGG01000000 [50]

Pseudomonas savastanoi pv. glycinea (Psg) race 4, sequence accession AEGH01000000 [50]

Pseudomonas sp. S9, sequence accession AFFX00000000 [51]

Pseudomonas stutzeri DSM4166, sequence accession CP002622 [52]

Pusillimonas sp. T7-7, sequence accession [53]

Rhizobium etli CNPAF512, sequence accession AEYZ00000000 [54]

Rhodobacter sphaeroides WS8N, sequence accession AFER00000000 [55]

Roseobacter sp. HTCC2038, sequence accession ABXE00000000 [56]

Rubrivivax benzoatilyticus JA2T, sequence accession AEWG00000000 [57]

Ruegeria TW15, sequence accession AEYW01000000 [58]

Salmonella enterica serovar Choleraesuis SCSA50, sequence accession CM001062CM001063 [59]

Salmonella enterica serovar Dublin SD3246, sequence accession CM001151CM001152 [59]

Salmonella enterica serovar Typhimurium 4/74, sequence accession CP002487 CP002490 [59]

Sphingomonas sp. S17, sequence accession AFGG01000000 [60]

Taylorella equigenitalis MCE9, sequence accession CP002456 [61]

Unnamed strain IMCC1989, sequence accession AEVK00000000 [62]

Unnamed strain IMCC2047, sequence accession AEGL00000000 [63]

Unnamed strain IMCC3088, sequence accession AEIG00000000 [64]

Unnamed strain IMCC9063 SAR11 subgroup 3, sequence accession CP002511 [65] 
Variovorax paradoxus S110, sequence accession [66]

Vibrio anguillarum pJM1, sequence accession AEZA00000000, AEZB00000000, AEZC00000000 [67]

Vibrio furnissii NCTC 11218, sequence accession CP002377 (chromosome I) and CP002378 (chromosome II) [68]

Vibrio parahaemolyticus clinical 04:K12 serotype, sequence accession AFBW00000000 [69]

Vibrio rotiferianus strain DAT722, sequence accession [70]

Vibrio vulnificus M06-24/0, sequence accession CP002469 and CP002470 [71]

Yersinia pestis KIM D27, sequence accession ADDC00000000 [72]

Yersinia enterocolitica 3/0:9, sequence accession CP002246 (chromosome)_CP002247 (pYV plasmid) [73]

Yersinia enterocolitica subsp. palearctica 2 serogroup 0:3, sequence accession FR729477 (chromosome) FR745874 (plasmid) [74]

\section{Phylum Firmicutes}

Bacillus amyloliquefaciens LL3, sequence accession CP002634, CP002635 [75]

Bacillus amyloliquefaciens TA208, sequence accession CP002627 [76]

Bacillus subtilis BSn5, sequence accession CP002468 [77]

Bacillus subtilis subsp. spizizenii gtP20b, sequence accession AEHM00000000 [78]

Bacillus thuringiensis YBT-020, sequence accession CP002508 (chromosome),

CP002509 (plasmid pBMB26), CP002510 (plasmid pBMB28 [79]

"Bacillus thuringiensis subsp. chinensis" CT-43, sequence accession CP001907.1, CP001910.1, CP001908.1, СР001915.1, CP001913.1, CP001911.1, CP001909.1, CP001917.1, CP001916.1, СР001914.1, CP001912.1 [80]

Caloramator australicus RC3T, sequence accession DRA000322 [81]

Carnobacterium sp. 17-4, sequence accession CP002563, CP002564 [82]

Cellulosilyticum lentocellum DSM 5427, sequence accession ADVF00000000 [83]

Clostridium acetobutylicum EA 2018, sequence accession NC_003030 [84]

Clostridium botulinum group III, sequence accession CP002410 - CP002415 [85]

Clostridium botulinum H04402 065, sequence accession FR773526 [86]

Clostridium thermocellum DSM1313, sequence accession CP002416 [87]

Enterococcus faecalis 62, sequence accession CP002491 - CP002495 [88]

Erysipelothrix rhusiopathiae ATCC 19414, sequence accession AP012027 [89]

Eubacterium limosum KIST612, sequence accession CP002273 [90]

Exiguobacterium sp. AT1b, sequence accession CP001615 [91]

"Halanaerobium hydrogenoforman", sequence accession CP002304 [92] 
Lactobacillus amylovorus GRL1118, sequence accession CP002338 [93]

Lactobacillus animalis KCTC 3501, sequence accession AEOF00000000 [94]

Lactobacillus buchneri NRRL B-30929, sequence accession CP002652, CP002653

(PLBU03), CP002654 (pLBU02), CP002655 (pLBU03) [95]

Lactobacillus casei (EP 164209630B1), sequence accession CP002616 and CP002617 [96]

Lactobacillus casei BD-II, sequence accession CP002618 and CP002619 [97]

Lactobacillus coryniformis subsp. coryniformis KCTC 3167, sequence accession AELK00000000 [98]

Lactobacillus delbrueckii subsp. bulgaricus, sequence accession CP000156 [99]

Lactobacillus delbrueckii subsp. bulgaricus ND02, sequence accession CP002341 and CP002342 [100]

Lactobacillus farciminis KCTC 3681, sequence accession AEOT00000000 [101]

Lactobacillus helveticus H10, sequence accession CP002429 (chromosome) and CP002430 (plasmid) [102]

Lactobacillus plantarum ST-III, sequence accession CP002222 [103]

Lactobacillus reuteri ATCC 53608, sequence accession CACS02000000 [104]

Lactococcus garvieae 21881, sequence accession AFCC01000000 [105]

Lactococcus garvieae UNIUD074, sequence accession AFHF01000000 [106]

Lactococcus lactis subsp. lactis CV56, sequence accession CP002365 through

CP002370 [107]

Leuconostoc fallax KCTC 3537, sequence accession AEIZ00000000 [108]

Leuconostoc gelidum KCTC 3527, sequence accession AEMI00000000 [109]

Leuconostoc inhae KCTC 3774, sequence accession AEMJ00000000 [110]

Listeria monocytogenes J1-220, sequence accession AFBU00000000 [111]

Listeria monocytogenes J1816, sequence accession AFBU00000000 [111]

Listeria monocytogenes HCC23, sequence accession CP001175 [112]

Melissococcus plutonius ATCC 35311, sequence accession AP012200 (chromosome), AP012201 (plasmid) [113]

Ornithinibacillus TW25, sequence accession AEWH00000000 [114]

Paenibacillus polymyxa SC2, sequence accession CP002213 (chromosome) and CP002214 (plasmid) [115]

Staphylococcus aureus 011, sequence accession AEUQ01000000 [116]

Staphylococcus aureus straub 046, sequence accession AEUR01000000 [116]

Staphylococcus aureus T0131, ST239-MRSA-SCCmec type III, sequence accession CP002643 [117]

Staphylococcus pseudintermedius ED99, sequence accession CP002478 [118] 
Staphylococcus pseudintermedius HKU10-03, sequence accession CP002439 [119]

Streptococcus parauberis KCTC11537BP, sequence accession CP002471 [120]

Streptococcus suis JS14, sequence accession CP002465 [121]

Streptococcus thermophilus ND03, sequence accession CP002340 [122]

Turicibacter sanguinis PC909, sequence accession ADMN00000000 [123]

Weissella cibaria KACC 11862, sequence accession AEKT01000000 [124]

\section{Phylum Tenericutes}

Mycoplasma alligatoris A21JP2T, sequence accession NZ_ADNC01000000 [125]

Mycoplasma crocodyli MP145T, sequence accession CP001991 [125]

Mycoplasma bovis PG45 (ATCC 25523), sequence accession CP002188 [126]

Mycoplasma haemofelis, sequence accession FR773153 [127]

Mycoplasma haemofelis Ohio2, sequence accession AEVA00000000 [128]

Mycoplasma suis Illinois, sequence accession ADWK01000001 [128]

Mycoplasma suis KI3806, sequence accession FQ790233 [129]

\section{Phylum Actinobacteria}

Bifidobacterium bifidum S17, sequence accession CP002220 [130]

Bifidobacterium longum subsp. longum BBMN68, sequence accession CP002286 [131]

Corynebacterium pseudotuberculosis I19, sequence accession CP002251 [132]

Janibacter sp. HTCC2649, sequence accession AAMN00000000 [133]

Microbacterium testaceum StLB037, sequence accession AP012052 [134]

Mycobacterium bovis BCG, sequence accession later [135]

Nocardioides sp. JS614, sequence accession CP000509, CP000508 [136]

Saccharopolyspora spinosa NRRL 18395, sequence accession AEYC00000000 [137]

Streptomyces griseoaurantiacus, sequence accession AEYX01000000 [138]

Streptomyces griseus XyelbKG-1, sequence accession ADFC00000000 [139]

Streptomyces sp. PP-C42, sequence accession AEWS01000000 [140]

Verrucosispora maris AB-18-032, sequence accession CP002638, CP002639 [141]

\section{Phylum Chlamydiae}

Chlamydia pecorum E58, sequence accession CP002608 [142]

Chlamydia psittaci 6BC, sequence accession CP002586 (chromosome), CP002587

(plasmid) [143]

Chlamydia psittaci Cal10, sequence accession AEZD00000000 [143]

Chlamydophila psittaci RD1, sequence accession FQ482149 (chromosome) FQ482150 (plasmid) [144] 


\section{Phylum Spirochaetes}

Borrelia burgdorferi, sequence accession ABJZ02000001-5 (chromosome) CP001519

(Ip17) CP001518 (IP28-2) CP001523 (Ip28-4) CP001524 (Ip54) CP001522 (for

cp26)_CP001517 (cp32-3) CP001520 (cp32-4) ABJZ02000006-7(Ip32-6) CP001521

(cp32-7)_CP001516 (cp32-12) [145]

Treponema paraluiscuniculi Cuniculi A, sequence accession CP002103 [146]

\section{Phylum Fibrobacteres}

Fibrobacter succinogenes S85 S85, sequence accession CP001792 [147]

\section{Phylum Bacteroidetes}

Algoriphagus sp. PR1, sequence accession AAXU01000000 [148]

Bacteroides vulgatus PC510, sequence accession ADK001000000 [149]

Kordia algicida OT-1, sequence accession ABIB00000000 [150]

Maribacter sp. HTCC2170, sequence accession CP002157 [151]

Riemerella anatipestifer RA-GD, sequence accession CP002562 [152]

Riemerella anatipestifer RA-YM, sequence accession AENH00000000 [153]

\section{Phylum Verrucomicrobia}

Akkermansia muciniphila ATCC BAA-835, sequence accession NC_010655 [154]

Opitutus terrae PB90-1, sequence accession CP001032 [155]

“Chthoniobacter flavus" Ellin428, sequence accession ABVL00000000 [156]

"Pedosphaera parvula" Ellin514, sequence accession ABOX00000000 [157]

\section{Phylum Lentisphaerae}

Victivallis vadensis ATCC BAA-548, sequence accession ABDE02000001-

ABDE02000027 [158]

\section{References}

1. Liu LJ, You XY, Zheng H, Wang S, Jiang CY, Liu SJ. Complete Genome Sequence of Metallosphaera cuprina, a Metal Sulfide-Oxidizing Archaeon from a Hot Spring. I Bacteriol 2011; 193:33873388. PubMed doi:10.1128/JB.05038-11

2. Mardanov AV, Gumerov VM, Beletsky AV, Prokofeva MI, Bonch-Osmolovskaya EA, Ravin NV, Skryabin KG. Complete genome sequence of the thermoacidophilic crenarchaeon Thermoproteus uzoniensis 768-20. J Bacteriol 2011.

3. Gumerov VM, Mardanov AV, Beletsky AV, Prokofeva MI, Bonch-Osmolovskaya EA, Ravin NV, Skryabin KG. Complete Genome Sequence of "Vulcanisaeta moutnovskia" Strain 768-28, a Novel Member of the Hyperthermophilic Crenarchaeal Genus Vulcanisaeta. I Bacteriol 2011; 193:2355-2356. PubMed doi:10.1128/JB.0023711
4. Barber RD, Zhang L, Harnack M, Olson MV, Kaul $\mathrm{R}$, Ingram-Smith C, Smith KS. Complete Genome Sequence of Methanosaeta concilii, a Specialist in Aceticlastic Methanogenesis. J Bacteriol 2011; 193:3668-3669. PubMed doi:10.1128//B.05031$\underline{11}$

5. Lee HS, Bae SS, Kim MS, Kwon KK, Kang SG, Lee $\mathrm{JH}$. Complete Genome Sequence of Hyperthermophilic Pyrococcus sp. Strain NA2, Isolated from a Deep-Sea Hydrothermal Vent Area. J Bacteriol 2011; 193:3666-3667. PubMed doi:10.1128/JB.05150-11

6. Vannier P, Marteinsson VT, Fridjonsson $\mathrm{OH}$, Oger P, Jebbar M. Complete Genome Sequence of the Hyperthermophilic, Piezophilic, Heterotrophic, and Carboxydotrophic Archaeon Thermococcus barophilus MP. I Bacteriol 2011; 193:1481-1482. PubMed doi:10.1128//B.01490$\underline{10}$ 
OW Nelson and GM Garrity

7. Kuznetsov BB, Ivanovsky RN, Keppen OI, Sukhacheva MV, Bumazhkin BK, Patutina EO, Beletsky AV, Mardanov AV, Baslerov RV, Panteleeva AN, et al. Draft Genome Sequence of the Anoxygenic Filamentous Phototrophic Bacterium Oscillochloris trichoides subsp. DG-6. J Bacteriol 2011;

193:321-322. PubMed doi:10.1128/JB.00931-10

8. Strnad H, Ridl J, Paces J, Kolar M, Vlcek C, Paces V. Complete Genome Sequence of the Haloaromatic Acid-Degrading Bacterium Achromobacter xylosoxidans A8. J Bacteriol 2011; 193:791-792. PubMed doi:10.1128//B.01299-10

9. Zarrilli R, Giannouli M, Rocco F, Loman NJ, Haines AS, Constantinidou C, Pallen MJ, Triassi M, Di Nocera PP. Genome Sequences of Three Acinetobacter baumannii Strains Assigned to the Multilocus Sequence Typing Genotypes ST2, ST25, and ST78. I Bacteriol 2011; 193:2359-2360. PubMed doi:10.1128//B.00245-11

10. Gao F, Wang Y, Liu YJ, Wu XM, Lv X, Gan YR, Song SD, Huang H. Genome Sequence of Acinetobacter baumannii MDR-TJ. J Bacteriol 2011; 193:2365-2366. PubMed doi:10.1128/JB.00226$\underline{11}$

11. Chen CC, Lin YC, Sheng WH, Chen YC, Chang SC, Hsia KC, Liao MH, Li SY. Genome Sequence of a Dominant, Multidrug-Resistant Acinetobacter baumannii Strain, TCDC-AB0715. I Bacteriol 2011; 193:2361-2362. PubMed doi:10.1128/]B.00244-11

12. Luo Y, Kong Q, Yang J, Golden G, Wanda SY, Jensen RV, Ernst PB, Curtiss R. Complete Genome Sequence of the Universal Killer, Salmonella enterica serovar Typhimurium UK-1 (ATCC 68169). J Bacteriol 2011..

13. Zhan $Y$, Yan $Y$, Zhang $W, Y u H$, Chen $M$, Lu W, Ping S, Peng Z, Yuan M, Zhou Z, et al. Genome Sequence of Acinetobacter calcoaceticus PHEA-2, Isolated from Industry Wastewater. I Bacteriol 2011; 193:2672-2673. PubMed doi:10.1128/|B.00261-11

14. Beatson SA, Luna M, Bachmann NL, Alikhan NF, Hanks KR, Sullivan MJ, Wee BA, Freitas-Almeida AC, dos Santos PA, de Melo JTB, et al. Genome Sequence of the Emerging Pathogen Aeromonas caviae. J Bacteriol 2011; 193:1286-1287. PubMed doi:10.1128/JB.01337-10

15. Li Y, Liu Y, Zhou Z, Huang H, Ren Y, Zhang Y, Li G, Zhou Z, Wang L. Complete Genome Sequence of Aeromonas veronii Strain B565. I Bacteriol
2011; 193:3389-3390.

PubMed doi:10.1128/JB.00347-11

16. Zhang $S, X u Y$, Zhou Z, Wang $S$, Yang R, Wang J, Wang $\mathrm{L}$. Complete genome sequence of B. pertussis CS, Chinese pertussis vaccine strain. J Bacteriol 2011.

17. Ding J, Pan $\mathrm{Y}$, Jiang $\mathrm{H}$, Cheng J, Liu $\mathrm{T}$, Qin $\mathrm{N}$, Yang Y, Cui B, Chen C, Liu C, et al. Whole Genome Sequences of Four Brucella Strains. J Bacteriol 2011; 193:3674-3675.

PubMed doi:10.1128//B.05155-11

18. Wang F, Hu S, Gao Y, Qiao Z, Liu W, Bu Z. Complete Genome Sequences of Brucella melitensis Strains M28 and M5-90, with Different Virulence Backgrounds. J Bacteriol 2011; 193:29042905. PubMed doi:10.1128/JB.00357-11

19. Seo YS, Lim JY, Choi BS, Kim H, Goo E, Lee B, Lim JS, Choi IY, Moon JS, Kim J, et al. Complete Genome Sequence of Burkholderia gladioli BSR3. J Bacteriol 2011

20. Weilharter A, Mitter B, Shin MV, Chain PSG, Nowak J, Sessitsch A. Complete Genome Sequence of the Plant Growth-Promoting Endophyte Burkholderia phytofirmans Strain PsJN. J Bacteriol 2011; 193:3383-3384.

PubMed doi:10.1128/JB.05055-11

21. Lackner G, Moebius N, Partida-Martinez L, Boland S, Hertweck C. Evolution of an endofungal Lifestyle: Deductions from the Burkholderia rhizoxinica Genome. BMC Genomics 2011; 12:210. PubMed doi:10.1186/1471-2164-12-210

22. Cooper KK, Cooper MA, Zuccolo A, Law B, Joens LA. Complete Genome Sequence of Campylobacter jejuni Strain S3. I Bacteriol 2011; 193:14911492. PubMed doi:10.1128//B.01475-10

23. Lin H, Lou B, Glynn J, Doddapaneni H, Civerolo E, Chen C, Duan Y, Zhou L, Vahling C. The Complete Genome Sequence of "Candidatus $\mathrm{Li}$ beribacter solanacearum", the Bacterium Associated with Potato Zebra Chip Disease. PLOS ONE 2011; 6:e19135.

PubMed doi:10.1371/journal.pone.0019135

24. Zheng Q, Zhang R, Jiao N. Genome Sequence of Citromicrobium Strain JLT1363, Isolated from the South China Sea. J Bacteriol 2011; 193:20742075. PubMed doi:10.1128//B.00121-11

25. Stephan R, Lehner A, Tischler P, Rattei T. Complete Genome Sequence of Cronobacter turicensis LMG 23827, a Food-Borne Pathogen Causing 
Deaths in Neonates. J Bacteriol 2011; 193:309-

310. PubMed doi:10.1128/JB.01162-10

26. Brown SD, Wall JD, Kucken AM, Gilmour CC, Podar M, Brandt CC, Teshima H, Detter JC, Han $\mathrm{CS}$, Land ML, et al. Genome Sequence of Mercury-Methylating and Pleomorphic Desulfovibrio africanus strain Walvis Bay. I Bacteriol 2011.

27. Brown SD, Gilmour CC, Kucken AM, Wall JD, Elias DA, Brandt CC, Podar M, Chertkov O, Held $B$, Bruce DC, et al. Genome Sequence of the Mercury-Methylating Strain Desulfovibrio desulfuricans ND132. J Bacteriol 2011; 193:2078-2079. PubMed doi:10.1128//B.00170-11

28. Glasner JD, Yang CH, Reverchon S, HugouvieuxCotte-Pattat N, Condemine G, Bohin JP, Van Gijsegem F, Yang S, Franza T, Expert D, et al. Genome Sequence of the Plant-Pathogenic Bacterium Dickeya dadantii 3937. I Bacteriol 2011; 193:2076-2077. PubMed doi:10.1128//B.01513$\underline{10}$

29. Zhu B, Zhang GQ, Lou MM, Tian WX, Li B, Zhou $X P$, Wang GF, Liu H, Xie GL, Jin GL. Genome Sequence of the Enterobacter mori Type Strain, LMG 25706, a Pathogenic Bacterium of Morus alba L. J Bacteriol 2011; 193:3670-3671.

PubMed doi:10.1128/JB.05200-11

30. Molina L, Rezzonico F, Defago G, Duffy B. Autoinduction in Erwinia amylovora: Evidence of an Acyl-Homoserine Lactone Signal in the Fire Blight Pathogen. J Bacteriol 2005; 187:3206-3213. PubMed doi:10.1128/JB.187.9.3206-3213.2005

31. Park DH, Thapa SP, Choi BS, Kim WS, Hur JH, Cho JM, Lim JS, Choi IY, Lim CK. Complete Genome Sequence of Japanese Erwinia Strain Ejp617, a Bacterial Shoot Blight Pathogen of Pear. I Bacteriol 2011; 193:586-587.

PubMed doi:10.1128//B.01246-10

32. Yi H, Cho YJ, Hur HG, Chun J. Genome Sequence of Escherichia coli AA86, Isolated from Cow Feces. J Bacteriol 2011; 193:3681.

PubMed doi:10.1128/JB.05193-11

33. Krause DO, Little AC, Dowd SE, Bernstein CN. Complete Genome Sequence of Adherent Invasive Escherichia coli UM146 Isolated from Ileal Crohn's Disease Biopsy Tissue. J Bacteriol 2011; 193:583. PubMed doi:10.1128/JB.01290-10

34. Johnson TJ, Fernandez-Alarcon C, Bojesen AM, Nolan LK, Trampel DW, Seemann T. Complete Genome Sequence of Gallibacterium anatis Strain UMN179, Isolated from a Laying Hen with Peri- tonitis. J Bacteriol 2011; 193:3676-3677.

PubMed doi:10.1128/JB.05177-11

35. Du XJ, Jia SR, Yang Y, Wang S. Genome Sequence of Gluconacetobacter sp. Strain SXCC-1, Isolated from Chinese Vinegar Fermentation Starter. J Bacteriol 2011; 193:3395-3396. PubMed doi:10.1128/JB.05147-11

36. Xu Z, Yue M, Zhou R, Fan Y, Bei W, Chen H. Genomic Characterization of Haemophilus parasuis SH0165, a Highly Virulent Strain of Serovar 5 Prevalent in China. PLoS ONE 2011; 6:e19631. PubMed doi:10.1371/journal.pone.0019631

37. Pedrosa F. Genome of Herbaspirillum seropedicae Strain SmR1, a Specialized Diazotrophic Endophyte of Tropical Grasses. PLoS Genet 2011; 7:e1002064. PubMed doi:10.1371/journal.pgen.1002064

38. Xiong $X \mathrm{H}$, Han $\mathrm{S}$, Wang J, Jiang Z, Chen W, Jia N, Wei $\mathrm{H}$, Cheng $\mathrm{H}$, Yang Y, Zhu B, et al. Complete Genome Sequence of the Bacterium Ketogulonicigenium vulgare Y25. I Bacteriol 2011; 193:315316. PubMed doi:10.1128//B.01189-10

39. Stein LY, Bringel F, DiSpirito AA, Han S, Jetten MSM, Kalyuzhnaya MG, Kits KD, Klotz MG, Op den Camp HJM, Semrau JD, et al. Genome Sequence of the Methanotrophic Alphaproteobacterium Methylocystis sp. Strain Rockwell (ATCC 49242). J Bacteriol 2011; 193:2668-2669. PubMed doi:10.1128//B.00278-11

40. Boden R, Ferriera S, Johnson J, Kelly DP, Murrell JC, Schafer H. Draft Genome Sequence of the Chemolithoheterotrophic, Halophilic Methylotroph Methylophaga thiooxydans DMS010. I Bacteriol 2011; 193:3154-3155.

PubMed doi:10.1128/JB.00388-11

41. Xiong $X \mathrm{H}$, Zhi J, Yang L, Wang J, Zhao $Y$, Wang $X$, Cui Z, Dong F, Li M, Yang YX, et al. Complete Genome Sequence of the Bacterium Methylovorus sp. Strain MP688, a High-Level Producer of Pyrroloquinolone Quinone. J Bacteriol 2011; 193:1012-1013. PubMed doi:10.1128//B.01431$\underline{10}$

42. Chen CC, Hsia KC, Huang CT, Wong WW, Yen MY, Li LH, Lin KY, Chen KW, Li SY. Draft Genome Sequence of a Dominant, MultidrugResistant Neisseria gonorrhoeae Strain, TCDCNG08107, from a Sexual Group at High Risk of Acquiring Human Immunodeficiency Virus Infection and Syphilis. J Bacteriol 2011; 193:17881789. PubMed doi:10.1128//B.00007-11 
OW Nelson and GM Garrity

43. Piet JR, Huis in 't Veld RAG, van Schaik BDC, van Kampen AHC, Baas F, van de Beek D, Pannekoek $\mathrm{Y}$, van der Ende A. Genome Sequence of Neisseria meningitidis Serogroup B Strain H44/76. J Bacteriol 2011; 193:2371-2372.

PubMed doi:10.1128//B.01331-10

44. Schoen C, Weber-Lehmann J, Blom J, Joseph B, Goesmann A, Strittmatter A, Frosch M. WholeGenome Sequence of the Transformable Neisseria meningitidis Serogroup A Strain WUE2594. J Bacteriol 2011; 193:2064-2065.

PubMed doi:10.1128/JB.00084-11

45. Oh HM, Kang I, Vergin KL, Lee K, Giovannoni SJ, Cho JC. Genome Sequence of Oceanicaulis sp. Strain HTCC2633, Isolated from the Western Sargasso Sea. J Bacteriol 2011; 193:317-318. PubMed doi:10.1128//B.01267-10

46. Li K, Wang S, Shi Y, Qu J, Zhai Y, Xu L, Xu Y, Song J, Liu L, Rahman MA, et al. Genome Sequence of Paracoccus sp. Strain TRP, a Chlorpyrifos Biodegrader. J Bacteriol 2011; 193:1786-1787. PubMed doi:10.1128/JB.00014-11

47. Oh HM, Kang I, Vergin KL, Kang D, Rhee KH, Giovannoni SJ, Cho JC. Complete Genome Sequence of Strain HTCC2503T of Parvularcula bermudensis, the Type Species of the Order "Parvularculales" in the Class Alphaproteobacteria. J

Bacteriol 2011; 193:305-306. PubMed doi:10.1128/]B.01205-10

48. Urbanczyk H, Ogura Y, Hendry TA, Gould AL, Kiwaki N, Atkinson JT, Hayashi T, Dunlap PV. Genome Sequence of Photobacterium mandapamensis svers. 1.1., Bioluminescent Symbiont of the Cardinalfish Siphamia versicolor. J Bacteriol 2011.

49. Li SG, Tang YQ, Nie Y, Cai M, Wu XL. Complete Genome Sequence of Polymorphum gilvum SL003B-26A1T, a Crude Oil-Degrading Bacterium from Oil-Polluted Saline Soil. I Bacteriol 2011; 193:2894-2895. PubMed doi:10.1128/|B.00333-11

50. Qi M, Wang D, Bradley C, Zhao Y. Genome Sequence Analyses of Pseudomonas savastanoi pv. glycinea and Subtractive Hybridization-Based Comparative Genomics with Nine Pseudomonads. PLOS ONE 2011; 6:e16451. PubMed doi:10.1371/journal.pone.0016451

51. Long M, Ruan L, Yu Z, Xu X. Genome sequence of Pseudomonas sp. S9, an extracellular arylsulfatase producing bacterium isolated from the mangrove soil. J Bacteriol 2011.
52. Yu H, Yuan M, Lu W, Yang J, Dai S, Li Q, Yang Z, Dong J, Sun L, Deng Z, et al. Complete Genome Sequence of the Nitrogen-fixing and Rhizosphereassociated Bacterium Pseudomonas stutzeri Strain DSM4166. J Bacteriol 2011.

53. Cao B, Ma T, Ren Y, Ren Y, Li G, Li P, Guo X, Ding P, Feng L. Complete Genome Sequence of Pusillimonas sp. T7-7: a cold-tolerate diesel oildegrading bacterium isolated from the Bohai Sea in China. J Bacteriol 2011.

54. Maarten F, Sanchez-Rodriguez A, Beullens S, Marchal K, Michiels J. Genome sequence of Rhizobium etli CNPAF512, a nitrogen-fixing symbiont isolated from bean root nodules in Brazil. J Bacteriol 2011.

55. Porter SL, Wilkinson DA, Byles ED, Wadhams $\mathrm{GH}$, Taylor S, Saunders NJ, Armitage JP: Genome Sequence of Rhodobacter sphaeroides strain WS8N. J. Bacteriol 2011.

56. Kang I, Vergin KL, Oh HM, Choi A, Giovannoni $\mathrm{SJ}$, Cho JC. Genome Sequence of Strain HTCC2083, a Novel Member of the Marine Clade Roseobacter. J Bacteriol 2011; 193:319-320. PubMed doi:10.1128//B.01268-10

57. Mohammed M, Isukapatla A, Mekala LP, Eedara Veera Venkata RP, Chintalapati S, Chintalapati VR. Genome Sequence of the Phototrophic Betaproteobacterium Rubrivivax benzoatilyticus Strain JA2 ${ }^{\top}$. I Bacteriol 2011; 193:2898-2899. PubMed doi:10.1128//B.00379-11

58. Lee J, Roh SW, Whon TW, Shin NR, Kim YO, Bae JW. Genome Sequence of Strain TW15, a Novel Member of the Genus Ruegeria, Belonging to the Marine Roseobacter Clade. I Bacteriol 2011; 193:3401-3402. PubMed doi:10.1128//B.0506711

59. Richardson EJ, Limaye B, Inamdar H, Datta A, Manjari KS, Pullinger GD, Thomson NR, Joshi RR, Watson M, Stevens MP. Genome sequences of Salmonella enterica serovar Typhimurium, Choleraesuis, Dublin and Gallinarum strains of highly defined virulence in food-producing animals. J

Bacteriol 2011; 193:3162-3163.

PubMed doi:10.1128/]B.00394-11

60. Farias ME, Revale S, Mancini E, Ordonez O, Turjanski A, Cortez N, Vazquez MP. Genome Sequence of Sphingomonas sp. S17, Isolated from an Alkaline, Hyperarsenic, and Hypersaline Volcano-Associated Lake at High Altitude in the Argentinean Puna. J Bacteriol 2011; 193:3686-3687. PubMed doi:10.1128/JB.05225-11 
61. Hébert L, Moumen B, Duquesne F, Breuil MF, Laugier C, Batto JM, Renault P, Petry S. Genome Sequence of Taylorella equigenitalis MCE9, the Causative Agent of Contagious Equine Metritis. J Bacteriol 2011; 193:1785.

PubMed doi:10.1128//B.01547-10

62. Jang Y, Oh HM, Kim H, Kang I, Cho JC. Genome Sequence of Strain IMCC1989, a Novel Member of the Marine Gammaproteobacteria. J Bacteriol 2011; 193:3672-3673. PubMed doi:10.1128/JB.05202-11

63. Kang I, Kang D, Oh HM, Kim H, Kim HJ, Kang TW, Kim SY, Cho JC. Genome Sequence of Strain IMCC2047, a Novel Marine Member of the Gammaproteobacteria. / Bacteriol 2011; 193:3688-3689. PubMed doi:10.1128/|B.05226$\underline{11}$

64. Jang Y, Oh HM, Kang I, Lee K, Yang SJ, Cho JC. Genome Sequence of Strain IMCC3088, a Proteorhodopsin-Containing Marine Bacterium Belonging to the OM60/NOR5 Clade. J Bacteriol 2011; 193:3415-3416. PubMed doi:10.1128/JB.05111-11

65. Oh HM, Kang I, Lee K, Jang Y, Lim SI, Cho JC. Complete Genome Sequence of Strain IMCC9063 Belonging to the SAR11 subgroup 3, Isolated from the Arctic Ocean. J Bacteriol 2011; 193:33793380. PubMed doi:10.1128/JB.05033-11

66. Han JI, Choi HK, Lee SW, Orwin PM, Kim J, LaRoe SL, Kim T, O'Neil J, Leadbetter JR, Lee SY, et al. Complete Genome Sequence of the Metabolically Versatile Plant Growth-Promoting Endophyte Variovorax paradoxus S110. I Bacteriol 2011; 193:1183-1190.

PubMed doi:10.1128//B.00925-10

67. Naka H, Dias GM, Thompson CC, Dubay C, Thompson FL, Crosa JH. Complete Genome Sequence of the Marine Fish Pathogen Vibrio anguillarum Harboring the pJM1 Virulence Plasmid and Genomic Comparison with Other Virulent Strains of V. anguillarum and V. ordalii. Infect Immun 2011; 79:2889-2900. PubMed doi:10.1128/IAI.05138-11

68. Lux TM, Lee R, Love J. Complete Genome Sequence of a Free-Living Vibrio furnissii sp. nov. Strain (NCTC 11218). J Bacteriol 2011; 193:14871488. PubMed doi:10.1128//B.01512-10

69. Gonzalez-Escalona N, Strain EA, De Jesus AJ, Jones JL, DePaola A. Genome Sequence of the Clinical O4:K12 Serotype Vibrio parahaemolyticus Strain 10329. J Bacteriol 2011; 193:34053406. PubMed doi:10.1128/JB.05044-11
70. Chowdhury PR, Boucher Y, Hassan KA, Paulsen IT, Stokes HW, Labbate M. Genome Sequence of Vibrio rotiferianus Strain DAT722. I Bacteriol

2011; 193:3381-3382.

PubMed doi:10.1128/JB.05089-11

71. Park JH, Cho YJ, Chun J, Seok YJ, Lee JK, Kim KS, Lee KH, Park SJ, Choi SH. Complete Genome Sequence of Vibrio vulnificus MO6-24/O. I Bacteriol 2011; 193:2062-2063.

PubMed doi:10.1128//B.00110-11

72. Losada L, Varga J, Hostetler J, Radune D, Kim M, Durkin S, Schneewind O, Nierman W. Genome Sequencing and Analysis of Yersina pestis KIM D27, an Avirulent Strain Exempt from Select Agent Regulation. PLoS ONE 2011; 6:e19054. PubMed doi:10.1371/journal.pone.0019054

73. Wang $X$, Li Y, Jing H, Ren Y, Zhou Z, Wang S, Kan B, Xu J, Wang L. Complete Genome Sequence of a Yersinia enterocolitica "Old World" (3/O:9) Strain and Comparison with the "New World" (1B/O:8) Strain. J Clin Microbiol 2011; 49:1251-1259. PubMed doi:10.1128/JCM.01921$\underline{10}$

74. Batzilla J, Hoper D, Antonenka U, Heesemann J, Rakin A. Complete Genome Sequence of Yersinia enterocolitica subsp. palearctica Serogroup O:3. I Bacteriol 2011; 193:2067.

PubMed doi:10.1128//B.01484-10

75. Geng W, Cao M, Song C, Xie H, Liu L, Yang C, Feng J, Zhang W, Jin Y, Du Y, et al. Complete Genome Sequence of Bacillus amyloliquefaciens LL3, Which Exhibits Glutamic Acid-Independent Production of Poly-\{gamma\}-Glutamic Acid. J Bacteriol 2011; 193:3393-3394. PubMed doi:10.1128/JB.05058-11

76. Zhang G, Deng A, Xu Q, Liang Y, Chen N, Wen T. Complete genome sequence of Bacillus amyloliquefaciens TA208, a strain for industrial production of guanosine and ribavirin. J Bacteriol 2011.

77. Deng Y, Zhu Y, Wang P, Zhu L, Zheng J, Li R, Ruan L, Peng D, Sun M. Complete Genome Sequence of Bacillus subtilis BSn5, an Endophytic Bacterium of Amorphophallus konjac with Antimicrobial Activity for the Plant Pathogen Erwinia carotovora subsp. carotovora. J Bacteriol 2011; 193:2070-2071. PubMed doi:10.1128//B.00129$\underline{11}$

78. Fan L, Bo S, Chen H, Ye W, Kleinschmidt K, Baumann HI, Imhoff JF, Kleine M, Cai D. Genome Sequence of Bacillus subtilis subsp. spizizenii gtP20b, Isolated from the Indian Ocean. J 
OW Nelson and GM Garrity

Bacteriol 2011; 193:1276-1277. PubMed doi:10.1128/JB.01351-10

79. Zhu Y, Shang H, Zhu Q, Ji F, Wang P, Fu J, Deng Y, Xu C, Ye W, Zheng J, et al. Complete Genome Sequence of Bacillus thuringiensis Serovar finitimus Strain YBT-020. J Bacteriol 2011; 193:23792380. PubMed doi:10.1128/JB.00267-11

80. He J, Wang J, Yin W, Shao X, Zheng H, Li M, Zhao Y, Sun M, Wang S, Yu Z. Complete Genome Sequence of Bacillus thuringiensis subsp. chinensis Strain CT-43. J Bacteriol 2011; 193:3407-3408. PubMed doi:10.1128/JB.05085$\underline{11}$

81. Ogg CD, Patel BKC. Draft Genome Sequence of Caloramator australicus Strain RC3T, a Thermoanaerobe from the Great Artesian Basin of Australia. J Bacteriol 2011; 193:2664-2665. PubMed doi:10.1128//B.00193-11

82. Voget S, Klippel B, Daniel R, Antranikian G. Complete Genome Sequence of Carnobacterium sp. 17-4. J Bacteriol 2011; 193:3403-3404. PubMed doi:10.1128//B.05113-11

83. Farias ME, Revale $\mathrm{S}$, Mancini E, Ordonez O, Turjanski A, Cortez N, Vazquez MP. Genome Sequence of Sphingomonas sp. S17, Isolated from an Alkaline, Hyperarsenic, and Hypersaline Volcano-Associated Lake at High Altitude in the Argentinean Puna. J Bacteriol 2011; 193:3686-3687. PubMed doi:10.1128/JB.05225-11

84. Hu S, Zheng H, Gu Y, Zhao J, Zhang W, Yang Y, Wang S, Guoping Z, Yang S, Jinag W. Comparative genomic and transcriptomic analysis revealed genetic characteristics related to solvent formation and xylose utilization in Clostridium acetobutylicum EA 2018. BMC Genomics 2011; 12:93. $\underline{\text { PubMed doi:10.1186/1471-2164-12-93 }}$

85. Skarin H, Hafstrom T, Westerberg J, Segerman B. Clostridium botulinum group III: a group with dual identity shaped by plasmids, phages and mobile elements. BMC Genomics 2011; 12:185. PubMed doi:10.1186/1471-2164-12-185

86. Carter AT, Pearson BM, Crossman LC, Drou N, Heavens D, Baker D, Febrer M, Caccamo M, Grant KA, Peck MW. Complete Genome Sequence of the Proteolytic Clostridium botulinum Type A5 (B3') Strain H04402 065. J Bacteriol 2011; 193:2351-2352. PubMed doi:10.1128/JB.00072-11

87. Feinberg L, Foden J, Barrett T, Davenport KW, Bruce D, Detter C, Tapia R, Han C, Lapidus A, Lucas $S$, et al. Complete Genome Sequence of the
Cellulolytic Thermophile Clostridium thermocelIum DSM1313. J Bacteriol 2011; 193:2906-2907. PubMed doi:10.1128/JB.00322-11

88. Brede DA, Snipen LG, Ussery DW, Nederbragt AJ, Nes IF. Complete Genome Sequence of the Commensal Enterococcus faecalis 62, Isolated from a Healthy Norwegian Infant. I Bacteriol 2011; 193:2377-2378.

PubMed doi:10.1128/JB.00183-11

89. Ogawa Y, Ooka T, Shi F, Ogura Y, Nakayama K, Hayashi T, Shimoji Y. The genome of Erysipelothrix rhusiopathiae, the causative agent of swine erysipelas, reveals new insights into the evolution of Firmicutes and its intracellular adaptations. J Bacteriol 2011.

90. Roh H, Ko HJ, Kim D, Choi DG, Park S, Kim S, Chang IS, Choi IG. Complete Genome Sequence of a Carbon Monoxide-Utilizing Acetogen, Eubacterium limosum KIST612. J Bacteriol 2011; 193:307-308. PubMed doi:10.1128/JB.01217-10

91. Vishnivetskaya TA, Lucas S, Copeland A, Lapidus A, Glavina del Rio T, Dalin E, Tice H, Bruce DC, Goodwin LA, Pitluck S, et al. Complete Genome Sequence of the Thermophilic Bacterium Exiguobacterium sp. AT1b. J Bacteriol 2011; 193:28802881. PubMed doi:10.1128/JB.00303-11

92. Brown SD, Begemann MB, Mormile MR, Wall JD, Han CS, Goodwin LA, Pitluck S, Land ML, Hauser LJ, Elias DA. Complete Genome Sequence of the Haloalkaliphilic, Hydrogen-Producing Bacterium Halanaerobium hydrogeniformans. J Bacteriol 2011; 193:3682-3683. PubMed doi:10.1128/JB.05209-11

93. Kant R, Paulin L, Alatalo E, de Vos WM, Palva A. Genome sequence of Lactobacillus amylovorus GRL1118, isolated from the pig ileum. J Bacteriol 2011.

94. Nam SH, Choi SH, Kang A, Kim DW, Kim RN, Kim A, Kim DS, Park HS. Genome Sequence of Lactobacillus animalis KCTC 3501. J Bacteriol 2011; 193:1280-1281.

PubMed doi:10.1128/JB.01505-10

95. Liu S, Leathers TD, Copeland A, Chertkov O, Goodwin L, Mills DA: Complete Genome Sequence of Lactobacillus buchneri NRRL B-30929, a Novel Strain from a Commercial Ethanol Plant. J. Bacteriol 2011.

96. Chen C, Ai L, Zhou F, Wang L, Zhang H, Chen W, Guo B. Complete genome sequence of probiotic Lactobacillus casei LC2W. J Bacteriol 2011. 
97. Ai L, Chen C, Zhou F, Wang L, Zhang H, Chen W, Guo B. Complete genome sequence of probiotic Lactobacillus casei BD-II. J Bacteriol 2011.

98. Nam SH, Choi SH, Kang A, Kim DW, Kim DS, Kim RN, Kim A, Park HS. Genome Sequence of Lactobacillus coryniformis subsp. coryniformis KCTC 3167. J Bacteriol 2011; 193:1014-1015. $\underline{\text { PubMed doi:10.1128/JB.01394-10 }}$

99. Hao P, Zheng H, Yu Y, Ding G, Gu W, Chen S, Yu Z, Ren S, Oda M, Konno T, et al. Complete Sequencing and Pan-Genomic Analysis of Lactobacillus delbrueckii subsp. bulgaricus Reveal Its Genetic Basis for Industrial Yogurt Production. PLOS ONE 2011; 6:e15964. $\underline{\text { PubMed doi:10.1371/journal.pone.0015964 }}$

100. Sun Z, Chen X, Wang J, Zhao W, Shao Y, Guo Z, Zhang X, Zhou Z, Sun T, Wang L, et al. Complete genome sequence of Lactobacillus delbrueckii subsp. bulgaricus strain ND02. J Bacteriol 2011; 193:3426-3427. PubMed doi:10.1128/JB.0500411

101. Nam SH, Choi SH, Kang A, Kim DW, Kim RN, Kim A, Kim DS, Park HS. Genome Sequence of Lactobacillus farciminis KCTC 3681. J Bacteriol 2011; 193:1790-1791. PubMed doi:10.1128//B.00003-11

102. Zhao W, Chen Y, Sun Z, Wang J, Zhou Z, Sun T, Wang L, Chen W, Zhang H. Complete Genome Sequence of Lactobacillus helveticus H10. J Bacteriol 2011; 193:2666-2667. PubMed doi:10.1128/JB.00166-11

103. Wang Y, Chen C, Ai L, Zhou F, Zhou Z, Wang L, Zhang H, Chen W, Guo B. Complete Genome Sequence of the Probiotic Lactobacillus plantarum ST-III. J Bacteriol 2011; 193:313-314.

PubMed doi:10.1128//B.01159-10

104. Heavens D, Tailford LE, Crossman L, Jeffers F, MacKenzie DA, Caccamo M, Juge N. Genome sequence of a vertebrate gut symbiont Lactobacillus reuteri ATCC 53608. J Bacteriol 2011.

105. Aguado-Urda M, Lopez-Campos GH, Blanco MM, Fernandez-Garayzabal JF, Cutuli MT, Aspiroz C, Lopez-Alonso V, Gibello A. Genome sequence of Lactococcus garvieae 21881, isolated from a case of human septicaemia. J Bacteriol 2011.

106. Reimundo P, Pignatelli M, Alcaraz LD, D'Auria G, Moya A, Guijarro JA. Genome Sequence of Lactococcus garvieae UNIUD074, Isolated in Italy from a Lactococcosis Outbreak. J Bacteriol
$2011 ; 193: 3684-3685$.

PubMed doi:10.1128/JB.05210-11

107. Gao Y, Lu Y, Teng KL, Chen ML, Zheng HJ, Zhu YQ, Zhong J. Complete Genome Sequence of Lactococcus lactis subsp. lactis CV56, a Probiotic Strain Isolated from the Vaginas of Healthy Women. J Bacteriol 2011; 193:2886-2887. $\underline{\text { PubMed doi:10.1128/JB.00358-11 }}$

108. Nam SH, Choi SH, Kang A, Kim DW, Kim DS, Kim RN, Kim A, Park HS. Genome Sequence of Leuconostoc fallax KCTC 3537. J Bacteriol 2011; 193:588-589. PubMed doi:10.1128/JB.01300-10

109. Kim DS, Choi SH, Kim DW, Kim RN, Nam SH, Kang A, Kim A, Park HS. Genome Sequence of Leuconostoc gelidum KCTC 3527, Isolated from Kimchi. J Bacteriol 2011; 193:799-800. PubMed doi:10.1128/JB.01371-10

110. Kim DS, Choi SH, Kim DW, Kim RN, Nam SH, Kang A, Kim A, Park HS. Genome Sequence of Leuconostoc inhae KCTC 3774, Isolated from Kimchi. J Bacteriol 2011; 193:1278-1279. PubMed doi:10.1128/JB.01458-10

111. Chen Y, Strain EA, Allard M, Brown EW. Genome Sequences of Listeria monocytogenes Strains J1816 and J1-220, Associated with Human Outbreaks. J Bacteriol 2011; 193:3424-3425. PubMed doi:10.1128/JB.05048-11

112. Steele CL, Donaldson JR, Paul D, Banes MM, Arick T, Bridges SM, Lawrence ML. Genome Sequence of Lineage III Listeria monocytogenes Strain HCC23. I Bacteriol 2011; 193:3679-3680. PubMed doi:10.1128/JB.05236-11

113. Okumura K, Arai R, Okura M, Kirikae T, Takamatsu D, Osaki M, Miyoshi-Akiyama T. Complete Genome Sequence of Melissococcus plutonius ATCC 35311. J Bacteriol 2011.

114. Whon TW, Roh SW, Shin NR, Kim MS, Kim YO, Bae JW. Genome Sequence of Strain TW25, a Novel Member of the Genus Ornithinibacillus in the Family Bacillaceae. J Bacteriol 2011; 193:2884-2885. PubMed doi:10.1128/JB.00308$\underline{11}$

115. Ma M, Wang C, Ding Y, Li L, Shen D, Jiang X, Guan D, Cao F, Chen H, Feng R, et al. Complete Genome Sequence of Paenibacillus polymyxa SC2, a Strain of Plant Growth-Promoting Rhizobacterium with Broad-Spectrum Antimicrobial Activity. J Bacteriol 2011; 193:311-312. PubMed doi:10.1128/JB.01234-10

116. Le Maréchal C, Hernandez D, Schrenzel J, Even S, Berkova N, Thiery R, Vautor E, Fitzgerald JR, 
OW Nelson and GM Garrity

Francois P, Le Loir Y. Genome Sequences of Two Staphylococcus aureus Ovine Strains That Induce Severe (Strain O11) and Mild (Strain O46) Mastitis. J Bacteriol 2011; 193:2353-2354.

PubMed doi:10.1128/JB.00045-11

117. Li Y, Cao B, Zhang Y, Zhou J, Yang B, Wang L. Complete Genome Sequence of Staphylococcus aureus T0131, an ST239-MRSA-SCCmec Type III Clone Isolated in China. J Bacteriol 2011; 193:3411-3412. PubMed doi:10.1128/JB.0513511

118. Zakour NLB, Bannoehr J, van den Broek AHM, Thoday KL, Fitzgerald JR. Complete Genome Sequence of the Canine Pathogen Staphylococcus pseudintermedius. I Bacteriol 2011; 193:23632364. PubMed doi:10.1128//B.00137-11

119. Tse H, Tsoi HW, Leung SP, Urquhart IJ, Lau SKP, Woo PCY, Yuen KY. Complete Genome Sequence of the Veterinary Pathogen Staphylococcus pseudintermedius Strain HKU10-03, Isolated in a Case of Canine Pyoderma. J Bacteriol 2011; 193:1783-1784. PubMed doi:10.1128//B.00023$\underline{11}$

120. Nho SW, Hikima JI, Cha IS, Park SB, Jang HB, del Castillo CS, Kondo H, Hirono I, Aoki T, Jung TS. Complete genome sequence and immunoproteomic analyses of the fish bacterial pathogen Streptococcus parauberis. I Bacteriol 2011; .

121. Hu P, Yang $M$, Zhang A, Wu J, Chen B, Hua $Y$, Yu J, Xiao J, Jin M. Complete Genome Sequence of Streptococcus suis Serotype 14 Strain JS14. J Bacteriol 2011; 193:2375-2376. PubMed doi:10.1128/JB.00083-11

122. Sun Z, Chen X, Wang J, Zhao W, Shao Y, Wu L, Zhou Z, Sun T, Wang L, Meng H, et al. Complete Genome Sequence of Streptococcus thermophilus Strain ND03. I Bacteriol 2011; 193:793-794. PubMed doi:10.1128//B.01374-10

123. Cuív PO, Klaassens ES, Durkin AS, Harkins DM, Foster L, McCorrison J, Torralba M, Nelson KE, Morrison M. Draft Genome Sequence of Turicibacter sanguinis PC909, Isolated from Human Feces. J Bacteriol 2011; 193:1288-1289. PubMed doi:10.1128/]B.01328-10

124. Kim DS, Choi SH, Kim DW, Nam SH, Kim RN, Kang A, Kim A, Park HS. Genome Sequence of Weissella cibaria KACC 11862. J Bacteriol 2011; 193:797-798. PubMed doi:10.1128/JB.01342-10

125. Brown DR, Farmerie WG, May M, Benders GA, Durkin AS, Hlavinka K, Hostetler J, Jackson J, Johnson J, Miller RH, et al. Genome Sequences of
Mycoplasma alligatoris A21JP2T and Mycoplasma crocodyli MP145 ${ }^{\top}$. J Bacteriol 2011; 193:28922893. PubMed doi:10.1128/JB.00309-11

126. Wise KS, Calcutt MJ, Foecking MF, Roske K, Madupu R, Methe BA. Complete Genome Sequence of Mycoplasma bovis Type Strain PG45 (ATCC 25523). Infect Immun 2011; 79:982-983. PubMed doi:10.1128/IAI.00726-10

127. Barker EN, Helps CR, Peters IR, Darby AC, Radford AD, Tasker S. Complete Genome Sequence of Mycoplasma haemofelis, a Hemotropic Mycoplasma. J Bacteriol 2011; 193:2060-2061. PubMed doi:10.1128/JB.00076-11

128. Messick JB, Santos AP, Guimaraes AMS. Complete Genome Sequences of Two Hemotropic Mycoplasmas, Mycoplasma haemofelis Strain Ohio2 and Mycoplasma suis Strain Illinois. J Bacteriol 2011; 193:2068-2069.

PubMed doi:10.1128/JB.00133-11

129. Guimaraes AM, Santo A, SanMiguel P, Walter T, Timenetsky J, Messick J. Complete Genome Sequence of Mycoplasma suis and Insights into Its Biology and Adaption to an Erythrocyte Niche. PLOS ONE 2011; 6:e19574. PubMed doi:10.1371/journal.pone.0019574

130. Zhurina D, Zomer A, Gleinser M, Brancaccio VF, Auchter M, Waidmann MS, Westermann C, van Sinderen D, Riedel CU. Complete Genome Sequence of Bifidobacterium bifidum S17. I Bacteriol 2011; 193:301-302. PubMed doi:10.1128/JB.01180-10

131. Hao Y, Huang D, Guo H, Xiao M, An H, Zhao L, Zuo F, Zhang B, Hu S, Song S, et al. Complete Genome Sequence of Bifidobacterium longum subsp. longum BBMN68, a New Strain from a Healthy Chinese Centenarian. J Bacteriol 2011; 193:787-788. PubMed doi:10.1128/JB.01213-10

132. Silva A, Schneider MPC, Cerdeira L, Barbosa MS, Ramos RTJ, Carneiro AR, Santos R, Lima M, D'Afonseca V, Almeida SS, et al. Complete Genome Sequence of Corynebacterium pseudotuberculosis 119, a Strain Isolated from a Cow in Israel with Bovine Mastitis. I Bacteriol 2011; 193:323-324. PubMed doi:10.1128/]B.01211-10

133. Thrash JC, Cho JC, Bertagnolli AD, Ferriera S, Johnson J, Vergin KL, Giovannoni SJ. Genome Sequence of the Marine Janibacter Sp. Strain HTCC2649. J Bacteriol 2011; 193:584-585. PubMed doi:10.1128/JB.01298-10

134. Morohoshi T, Wang WZ, Someya N, Ikeda T. Genome Sequence of Microbacterium testaceum 
StLB037, an N-Acylhomoserine Lactone-

Degrading Bacterium Isolated from Potato Leaves.

J Bacteriol 2011; 193:2072-2073.

PubMed doi:10.1128/JB.00180-11

135. Pan Y, Yang X, Duan J, Lu N, Leung AS, Tran V, Hu Y, Wu N, Liu D, Wang Z, et al. WholeGenome Sequences of Four Mycobacterium bovis BCG Vaccine Strains. I Bacteriol 2011; 193:31523153. PubMed doi:10.1128/JB.00405-11

136. Coleman NV, Wilson NL, Barry K, Brettin TS, Bruce DC, Copeland A, Dalin E, Detter JC, del Rio TG, Goodwin LA, et al. Genome Sequence of the Ethene- and Vinyl Chloride-Oxidizing Actinomycete Nocardioides sp. Strain JS614. I Bacteriol 2011; 193:3399-3400. PubMed doi:10.1128//B.05109-11

137. Pan Y, Yang X, Li J, Zhang R, Hu Y, Zhou Y, Wang J, Zhu B. The genome sequence of spinosyns-producing bacterium Saccharopolyspora spinosa NRRL 18395. J Bacteriol 2011

138. Li F, Jiang P, Zheng H, Wang S, Zhao G, Qin S, Liu Z. Draft Genome Sequence of the Marine Bacterium Streptomyces griseoaurantiacus M045, Which Produces Novel Manumycin-Type Antibiotics with a pABA Core Component. J Bacteriol 2011; 193:3417-3418.

PubMed doi:10.1128/JB.05053-11

139. Grubbs KJ, Biedermann PHW, Suen G, Adams SM, Moeller JA, Klassen JL, Goodwin LA, Woyke T, Munk AC, Bruce D, et al. Genome Sequence of Streptomyces griseus Strain XylebKG-1, an Ambrosia Beetle-Associated Actinomycete. J Bacteriol 2011; 193:2890-2891.

PubMed doi:10.1128/JB.00330-11

140. Fan L, Liu Y, Li Z, Baumann HI, Kleinschmidt K, Ye W, Imhoff JF, Kleine M, Cai D. Draft Genome Sequence of the Marine Streptomyces sp. Strain PP-C42, Isolated from the Baltic Sea. I Bacteriol 2011; 193:3691-3692.

PubMed doi:10.1128/JB.05097-11

141. Roh H, Uguru GC, Ko HJ, Kim S, Kim BY, Goodfellow M, Bull AT, Kim KH, Bibb MJ, Choi IG, et al. Genome Sequence of the Abyssomicin- and Proximicin-Producing Marine Actinomycete Verrucosispora maris AB-18-032. J Bacteriol 2011; 193:3391-3392. PubMed doi:10.1128//B.0504111

142. Mojica S, Huot Creasy H, Daugherty S, Read TD, Kim T, Kaltenboeck B, Bavoil P, Myers GSA. Genome Sequence of the Obligate Intracellular Animal Pathogen Chlamydia pecorum E58. I Bacte- riol 2011; 193:3690.

PubMed doi:10.1128/JB.00454-11

143. Grinblat-Huse V, Drabek EF, Huot Creasy H, Daugherty SC, Jones KM, Santana-Cruz I, Tallon LJ, Read TD, Hatch TP, Bavoil P, et al. Genome sequences of the zoonotic pathogens Chlamydia psittaci 6BC and Cal10. J Bacteriol 2011.

144. Seth-Smith HMB, Harris SR, Rance R, West AP, Severin JA, Ossewaarde JM, Cutcliffe LT, Skilton RJ, Marsh P, Parkhill J, et al. Genome Sequence of the Zoonotic Pathogen Chlamydophila psittaci. J

Bacteriol 2011; 193:1282-1283. PubMed doi:10.1128//B.01435-10

145. Gorbacheva VY, Godfrey HP, Cabello FC. Analysis of the bmp Gene Family in Borrelia burgdorferi Sensu Lato. J Bacteriol 2000; 182:2037-2042. PubMed doi:10.1128//B.182.7.2037-2042.2000

146. Šmajs D, Zobaníková M, Strouhal M, Čejková D, Dugan-Rocha S, Pospíšilová P, Norris S, Albert T, Qin X, Hallsworth-Pepin K, et al. Complete Genome Sequence of Treponema paraluiscuniculi, Strain Cuniculi A: The Loss of Infectivity to Humans Is Associated with Genome Decay. PLoS ONE 2011; 6:e20415. PubMed doi:10.1371/journal.pone.0020415

147. Suen G, Weimer P, Stevenson D, Aylward F, Boyum J, Deneke J, Colleen D, Ivanova N, Mikhailova N, Chertkov O, et al. The Complete Genome Sequence of Fibrobacter succinogenes S85 Reveals a Cellulolytic and Metabolic Specialist. PLOS ONE 2011; 6:e18814. PubMed doi:10.1371/journal.pone.0018814

148. Alegado RA, Ferriera S, Nusbaum C, Young SK, Zeng Q, Imamovic A, Fairclough SR, King N. Complete Genome Sequence of Algoriphagus sp. PR1, Bacterial Prey of a Colony-Forming Choanoflagellate. J Bacteriol 2011; 193:1485-1486. PubMed doi:10.1128/JB.01421-10

149. Cuiv P, Klaassens ES, Durkin AS, Harkins DM, Foster L, McCorrison J, Torralba M, Nelson KE, Morrison M. The draft genome sequence of Bacteroides vulgatus PC510, a strain isolated from human faeces. J Bacteriol 2011.

150. Lee HS, Kang SG, Kwon KK, Lee JH, Kim SJ. Genome Sequence of the Algicidal Bacterium Kordia algicida OT-1. J Bacteriol 2011.

151. Oh HM, Kang I, Yang SJ, Jang Y, Vergin KL, Giovannoni SJ, Cho JC. Complete Genome Sequence of Strain HTCC2170, a Novel Member of the Genus Maribacter in the Family Flavobacte- 
riaceae. J Bacteriol 2011; 193:303-304.

PubMed doi:10.1128/JB.01207-10

152. Yuan J, Liu W, Sun M, Song S, Cai J, Hu S. Complete Genome Sequence of the Pathogenic Bacterium Riemerella anatipestifer Strain RA-GD. J Bacteriol 2011; 193:2896-2897.

PubMed doi:10.1128/JB.00301-11

153. Zhou Z, Peng X, Xiao Y, Wang X, Guo Z, Zhu L, Liu M, Jin H, Bi D, Li Z, et al. Genome Sequence of Poultry Pathogen Riemerella anatipestifer Strain RA-YM. J Bacteriol 2011; 193:1284-1285.

PubMed doi:10.1128/JB.01445-10

154. van Passel MW, Kant R, Zoetendal E, Plugge C, Derrien M, Malfatti S, Chain P, Woyke T, de Vos W, Smidt H. The Genome of Akkermansia muciniphila, a Dedicated Intestinal Mucin Degrader, and Its Use in Exploring Intestinal Metagenomes. PLOS ONE 2011; 6:e16876. PubMed doi:10.1371/journal.pone.0016876

155. van Passel MWJ, Kant R, Palva A, Copeland A, Lucas S, Lapidus A, Glavina del Rio T, Pitluck S, Goltsman E, Clum A, et al. Genome Sequence of the Verrucomicrobium Opitutus terrae PB90-1, an Abundant Inhabitant of Rice Paddy Soil Ecosys- tems. J Bacteriol 2011; 193:2367-2368.

PubMed doi:10.1128/JB.00228-11

156. Kant R, van Passel MWJ, Palva A, Lucas S, Lapidus A, Glavina del Rio T, Dalin E, Tice H, Bruce D, Goodwin L, et al. Genome Sequence of Chthoniobacter flavus Ellin428, an Aerobic Heterotrophic Soil Bacterium. J Bacteriol 2011; 193:2902-2903. PubMed doi:10.1128/JB.00295$\underline{11}$

157. Kant R, van Passel MWJ, Sangwan P, Palva A, Lucas S, Copeland A, Lapidus A, Glavina del Rio T, Dalin E, Tice H, et al. Genome Sequence of "Pedosphaera parvula" Ellin514, an Aerobic Verrucomicrobial Isolate from Pasture Soil. I Bacteriol 2011; 193:2900-2901. PubMed doi:10.1128/JB.00299-11

158. van Passel MWJ, Kant R, Palva A, Lucas S, Copeland A, Lapidus A, Glavina del Rio T, Dalin E, Tice $H$, Bruce D, et al. Genome Sequence of Victivallis vadensis ATCC BAA-548, an Anaerobic Bacterium from the Phylum Lentisphaerae, Isolated from the Human Gastrointestinal Tract. J Bacteriol 2011; 193:2373-2374. PubMed doi:10.1128/JB.00271-11 